\title{
Invasive fungal tracheobronchitis in mechanically ventilated critically ill patients: underlying conditions, diagnosis, and outcomes
}

\author{
Chun-Yu Lin ${ }^{1,3+}$, Wei-Lun Liu, ${ }^{4,5,6+}$, Che-Chia Chang ${ }^{7}$, Hou-Tai Chang ${ }^{8}$, Han-Chung Hu, ${ }^{2,3}$, Kuo-chin Kao ${ }^{2,3}$, \\ Ning-Hung Chen ${ }^{2,3}$, Ying-Jen Chen ${ }^{1,3}$, Cheng-Ta Yang ${ }^{2,3}$, Chung-Chi Huang ${ }^{2,3^{*}}$ and George Dimopoulos ${ }^{9}$
}

\begin{abstract}
Background: Invasive fungal tracheobronchitis (IFT) is a severe form of pulmonary fungal infection that is not limited to immunocompromised patients. Although respiratory failure is a crucial predictor of death, information regarding IFT in critically ill patients is limited.

Methods: In this retrospective, multicenter, observational study, we enrolled adults diagnosed as having IFT who had been admitted to the intensive care unit between January 2007 and December 2015. Their demographics, clinical imaging data, bronchoscopic and histopathological findings, and outcomes were recorded.

Results: This study included 31 patients who had been diagnosed as having IFT, comprising 24 men and 7 women with a mean age of $64.7 \pm 13.7$ years. All patients developed respiratory failure and received mechanical ventilation before diagnosis. Eighteen (58.1\%) patients had diabetes mellitus, and 12 (38.7\%) had chronic lung disease. Four (12.9\%) patients had hematologic disease, and none of the patients had neutropenia. Twenty-five (80.6\%) patients were diagnosed as having proven IFT, and the remaining patients had probable IFT. Aspergillus spp. (61.3\%) were the most common pathogenic species, followed by Mucorales (25.8\%) and Candida spp. (6.5\%). The diagnoses in six (19.4\%) patients were confirmed only through bronchial biopsy and histopathological examination, whereas their cultures of bronchoalveolar lavage fluid were negative for fungi. The overall in-hospital mortality rate was 93.5\%.

Conclusions: IFT in critically ill patients results in a high mortality rate. Diabetes mellitus is the most prevalent underlying disease, followed by chronic lung disease. In addition to Aspergillus spp., Mucorales is another crucial pathogenic species. Bronchial lesion biopsy is the key diagnostic strategy.
\end{abstract}

Keywords: Invasive fungal tracheobronchitis, Aspergillosis, Mucormycosis, Critical care, Outcome

\section{Background}

Invasive fungal disease is a life-threatening disease that mostly occurs in immunocompromised patients. The incidence of pulmonary fungal infection has dramatically increased in recent years [1]. Aspergillus spp. is the most common pathogenic species among pulmonary fungal infection $[2,3]$. The overall mortality rate of invasive aspergillosis is approximately 50\% [4-6]. Moreover, the

\footnotetext{
*Correspondence: cch4848@cloud.cgmh.org.tw

${ }^{\dagger}$ Chun-Yu Lin and Wei-Lun Liu contributed equally to the work

${ }^{2}$ Department of Pulmonary and Critical Care Medicine, Chang Gung

Memorial Hospital at Linkou, Taoyuan, Taiwan

Full list of author information is available at the end of the article
}

frequency of invasive fungal infections caused by nonAspergillus filamentous fungi is also increasing, and these infections are associated with devastating outcomes similar to that of invasive aspergillosis [5]. In addition to patients with conventional risk factors including neutropenia and those who have undergone stem cell transplantations, patients with chronic obstructive pulmonary disease, chronic renal failure, and liver cirrhosis may develop invasive fungal infections $[2,7,8]$. Critically ill patients who are admitted to intensive care units (ICUs) have been increasingly recognized as a population at a particularly high risk of pulmonary fungal infection [2]. Moreover, invasive aspergillosis in critically ill patients 
without malignancy who receive mechanical ventilation results in very poor outcomes and a mortality rate of $90 \%$ [9].

Invasive fungal tracheobronchitis (IFT) is a rare but severe form of pulmonary fungal infection that has been increasingly observed in critically ill patients [2, 10]. Diagnosing IFT is considerably difficult because of the nonspecific clinical manifestations and the low yields in microbiological tests $[2,11,12]$. The mortality rate of IFT caused by different fungi varies from 20 to $80 \%$ [11, 1316]. Patients with Aspergillus tracheobronchitis who have developed acute respiratory failure exhibit substantially poorer outcomes than those without respiratory distress do (mortality rate $69.2-93.8$ vs. $25-32.8 \%$ ) $[11,15,16]$. Moreover, ICU admission is a strong predictor of death in patients with non-Aspergillus mold invasive infections [5]. However, information regarding IFT in critically ill patients is limited.

The aim of the current study is to evaluate the diagnostic approach and the outcomes of IFT in critically ill patients.

\section{Methods \\ Study design and subjects}

In this retrospective, multicenter, observational study, we included critically ill adult patients with IFT who had been admitted to medical ICUs between January 2007 and December 2015 at the Linkou and Chiayi branches of Chang Gung Memorial Hospital, Far Eastern Memorial Hospital, and the Liouying branch of Chi Mei Medical Center. This study was approved by the institutional review boards of Chang Gung Memorial Hospital (CGMH 104-7452B). The patients were classified as having proven or probable IFT by using the revised definitions for invasive fungal infections from the European Organization for the Research and Treatment of Cancer/ Mycosis Study Group (EORTC/MSG) [17]. Histopathology was used to diagnose proven IFT. Probable IFT refers to the presence of positive cultures for fungal species from bronchoalveolar lavage (BAL) specimens accompanied by tracheobronchitis. All patients underwent fiberoptic bronchoscopy. From the bronchoscopic findings, IFT was classified into pseudomembranous, ulcerative, or obstructive forms according to Denning's classification [18]. The patients' demographic data; underlying diseases; clinical presentation; disease severity; laboratory parameters; bronchoscopic, microbiological, and histopathological findings; medications; and outcomes were recorded.

Overall in-hospital mortality was assessed. If the study patients were alive, survival was recorded until the date they were lost to follow-up or the date the study concluded. Because of the retrospective, observational nature of this study and the lack of any modification in the general management of the patients, the need for informed consent was waived.

\section{Statistical analyses}

All statistical analyses were performed using GraphPad Prism statistical software (GraphPad Prism, version 5.01). The categorical variables are presented as counts (percentages), and the continuous variables are presented as the means \pm standard deviations.

\section{Results}

This study included 31 critically ill patients who had been diagnosed as having IFT, comprising 24 men and 7 women with a mean age of $64.7 \pm 13.7$ years. Table 1 summarizes the demographics and underlying conditions of the patients who were hospitalized in the medical ICU for IFT. Thirty (96.8\%) patients had underlying diseases. Only one patient, who was a light smoker, had no medical history. Diabetes mellitus (DM; 18 patients, [58.1\%]) was the most predominant underlying condition in the IFT patients. The median HbA1c level was 8.1\% (5.4-13\%). Five patients were newly diagnosed as having DM. Nine patients were taking oral anti-diabetic agents. Four patients had received insulin therapy. Three of these DM patients had proteinuria and chronic renal disease. Chronic lung disease (12 patients [38.7\%]) was the second most predominant underlying disease. Four patients (12.9\%) had solid organ tumors, and four (12.9\%) had hematologic disease. None of these IFT patients had chronic renal failure or neutropenia, and none had undergone solid organ transplantations. Moreover, 17 patients $(54.8 \%)$ had received systemic steroid treatment before diagnosis. Three patients (9.7\%) developed IFT after being diagnosed as having H1N1 pneumonia. The overall in-hospital mortality rate was $93.5 \%$ (29 patients). The median time spent in the ICU before diagnosis was 5 days (0-19 days). The median length of ICU stay was 14 days (2-85 days). The median survival time after diagnosis was 10 days ( $0-85$ days). Table 2 summarizes the clinical manifestations of the critically ill patients with IFT. The mean Acute Physiology and Chronic Health Evaluation II (APACHE II) score on ICU admission was $23.1 \pm 10.4$. Because of respiratory failure, all patients received mechanical ventilation before diagnosis. Furthermore, 21 patients (67.7\%) underwent computed tomography (CT). Consolidation was the most frequent finding (19 patients [61.3\%]). Only one patient exhibited the air crescent sign on CT, and none of the patients had the halo sign. All patients underwent bronchoscopies and BAL. Moreover, 27 and 12 patients ( 87.1 and $38.7 \%$ ) had the pseudomembranous and ulcerative forms of IFT, respectively. The obstructive form (4 patients [12.9\%]) was the least frequent form of IFT (Table 2). Bronchial biopsy was performed in 27 
Table 1 Demographics and underlying conditions of 31 patients with invasive fungal tracheobronchitis

\begin{tabular}{ll}
\hline Variable & No. of patients (\%) \\
\hline Age, years (mean \pm SD) & $64.7 \pm 13.7$ \\
Gender, male & $24(77.4)$ \\
Current/ex-smoker & $15(48.4)$ \\
Underlying disease & $30(96.8)$ \\
DM & $18(58.1)$ \\
Chronic lung disease & $12(38.7)$ \\
COPD/asthma & $8(25.8)$ \\
Old TB & $2(6.5)$ \\
Bronchiectasis & $2(6.5)$ \\
Solid organ cancer & $4(12.9)$ \\
Hematologic disease & $4(12.9)$ \\
Liver cirrhosis & $3(9.7)$ \\
Systemic steroids before diagnosis & $17(54.8)$ \\
Duration of steroids before ICU admission, day, & $49(14-90)$ \\
$\quad$ median (IQR) & \\
Daily dosage of steroids, mg, median (IQR) & $50(29-71)$ \\
Inhaled corticosteroids before diagnosis & $3(9.7)$ \\
H1N1 infection before diagnosis & $3(9.7)$ \\
\hline
\end{tabular}

$S D$ standard deviation, $D M$ diabetes mellitus, COPD chronic obstructive pulmonary disease, $T B$ tuberculosis, ICU intensive care unit, IQR interquartile range

patients (87\%). Biopsy was not performed in the remaining four patients $(12.9 \%)$ because of the extremely low platelet count $\left(<5000\right.$ per $\left.\mathrm{mm}^{3}\right)$ or severe hypoxemia under ventilator support (fraction of inspired oxygen 100\%). None of the patients had developed massive hemoptysis or hypoxemia after the bronchial biopsy. Figure 1 illustrates the distinct histological images and indistinguishable bronchoscopic and radiographic findings of IFT caused by different fungal species. The mortality rate did not differ significantly between the patients with proven and probable IFT $(96 \%$ [24 of 25] vs. 83.3\% [5 of 6]; $p=0.35$ ).

In this study, Aspergillus spp. was found to be the most common pathogenic species of IFT (19 patients [61.3\%]), followed by Mucorales (8 patients [25.8\%]). Candida spp. was the causative pathogen of IFT in two patients (6.5\%). A further two patients were infected by undifferentiated mold (Table 2). The galactomannan antigen levels were evaluated in 16 of the 19 patients with Aspergillus tracheobronchitis and in 4 of the 12 patients with non-Aspergillus-related fungal tracheobronchitis. In patients with Aspergillus tracheobronchitis, the galactomannan antigen levels in the serum and BAL were $3.44 \pm 2.8$ and $4.87 \pm 2.6$, respectively. In patients with non-Aspergillus-related fungal tracheobronchitis, the galactomannan antigen levels were less than 0.5 . The two survivors were infected by Aspergillus spp. and undifferentiated mold. The mortality rate did
Table 2 Clinical manifestations of 31 patients with invasive fungal tracheobronchitis

\begin{tabular}{|c|c|}
\hline Variable & No. of patients (\%) \\
\hline APACHE II score on ICU admission, mean \pm SD & $23.1 \pm 10.4$ \\
\hline AKI requiring $R R T$ & $14(45.2)$ \\
\hline RF before diagnosis reached & $31(100)$ \\
\hline Time in the ICU before diagnosis, days (IQR) & $5(1.8-8)$ \\
\hline Length of ICU stay, days (IQR) & $14(8-27)$ \\
\hline Concurrent bacterial sepsis & $18(58.1)$ \\
\hline Parenchymal involvement & $31(100)$ \\
\hline CT scan & $21(67.7)$ \\
\hline Consolidation & $19(61.3)$ \\
\hline Cavitation & $4(12.9)$ \\
\hline Air crescent sign & $1(3.2)$ \\
\hline \multicolumn{2}{|l|}{ Bronchoscopic classification } \\
\hline Pseudomembranous & $27(87.1)$ \\
\hline Ulcerative & $12(38.7)$ \\
\hline Obstructive & $4(12.9)$ \\
\hline \multicolumn{2}{|l|}{ Diagnosis of IFT } \\
\hline Proven & 25 (80.6) \\
\hline Probable & $6(19.4)$ \\
\hline \multicolumn{2}{|l|}{ Pathogen } \\
\hline Aspergillus spp. & $19(61.3)$ \\
\hline Aspergillus fumigatus & $11(35.4)$ \\
\hline Aspergillus flavus & $2(6.5)$ \\
\hline Aspergillus terrus & $1(3.2)$ \\
\hline Undifferentiated Aspergillus species & $5(16.1)$ \\
\hline Mucorales & $8(25.8)$ \\
\hline Candida spp. & $2(6.5)$ \\
\hline Undifferentiated mold & $2(6.5)$ \\
\hline \multicolumn{2}{|l|}{ BAL fungal culture } \\
\hline Positive & 25 (80.6) \\
\hline Negative & $6(19.4)$ \\
\hline \multicolumn{2}{|c|}{ Galactomannan level in Aspergillus tracheobronchitis } \\
\hline Serum (index) & $3.44 \pm 2.8$ \\
\hline BAL (index) & $4.87 \pm 2.6$ \\
\hline Antifungal therapy & $29(93.5)$ \\
\hline Voriconazole & $15(48.4)$ \\
\hline Echinocandin & $19(61.3)$ \\
\hline Combination therapy & $8(25.8)$ \\
\hline
\end{tabular}

APACHE II Acute Physiology and Chronic Health Evaluation II, ICU intensive care unit, $S D$ standard deviation, $A K I$ acute kidney injury, $R R T$ renal replacement therapy, $R F$ respiratory failure, $I Q R$ interquartile range, $C T$ computed tomography, IFT invasive fungal tracheobronchitis, BAL bronchoalveolar lavage

not differ among patients with IFT caused by different fungal species. Only nine patients (29\%) had positive fungal culture results for lower respiratory tract specimens before bronchoscopy. Six patients (19.4\%) had negative fungal culture results for BAL fluid after bronchoscopy. They were diagnosed through histopathology of bronchial biopsy specimens. 

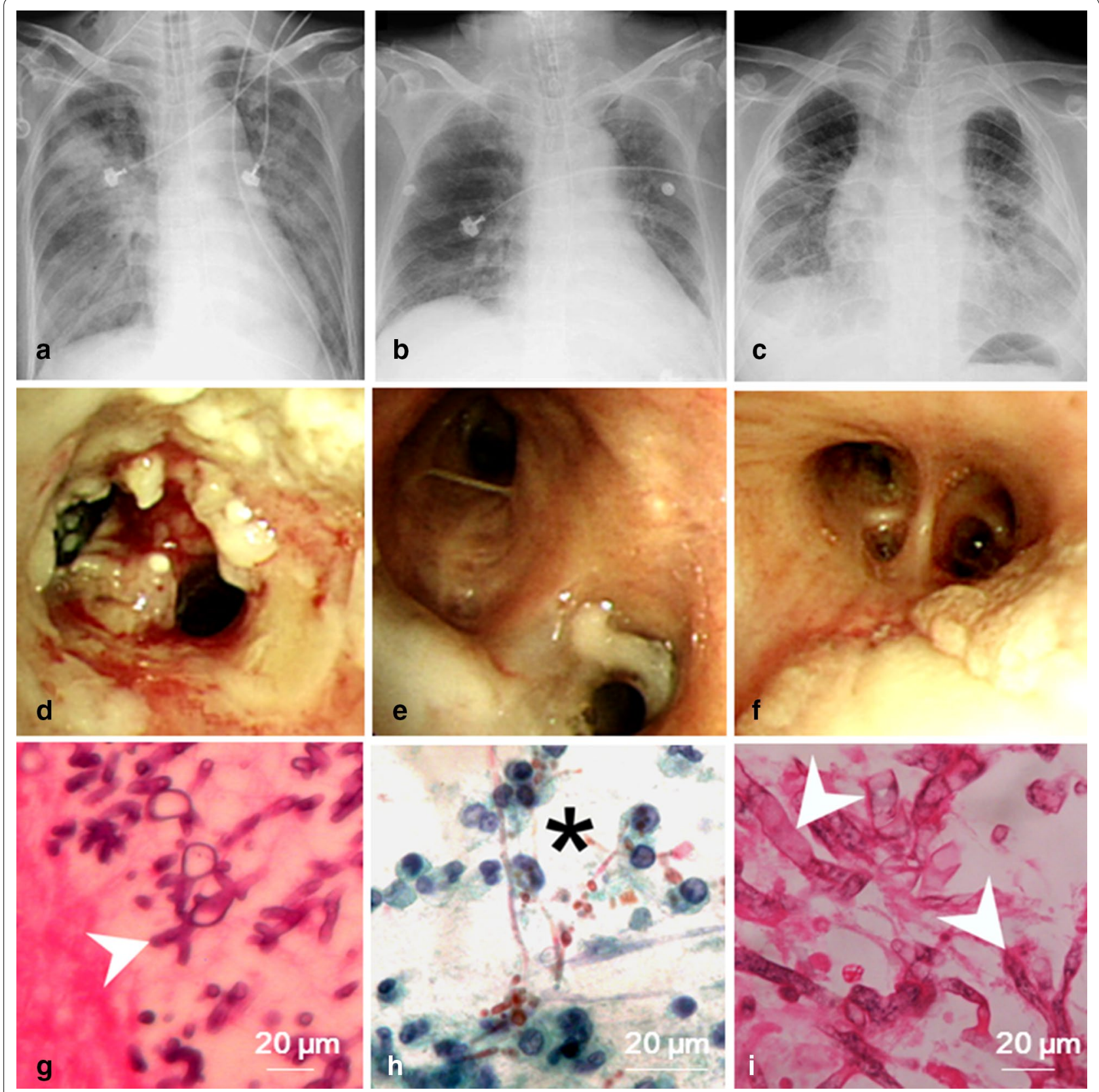

Fig. 1 Chest radiograph and bronchoscopic and histological examinations of IFT. a Invasive Aspergillus tracheobronchitis. b Invasive Candida tracheobronchitis. c Mucorales-related invasive tracheobronchitis. d Bronchoscopic view of invasive Aspergillus tracheobronchitis. e Bronchoscopic view of invasive Candida tracheobronchitis. $\mathbf{f}$ Bronchoscopic view of Mucorales-related invasive tracheobronchitis. $\mathbf{g}$ Histopathological examination revealed septate fungal hyphae branching at a $45^{\circ}$ angle (arrowhead), which is characteristic of Aspergillus spp. (magnification: $400 \times$ ). $\mathbf{h}$ Histopathological examination revealed yeast cells and pseudohyphae (star), which are characteristic of Candida spp. (magnification: 400x). i Histopathological examination revealed broad, thin-walled, non-septate hyphae, which are characteristic of Mucormycete (left arrowhead) and the other septate fungal hyphae branching at a sharp angle, which are characteristic of Aspergillus spp. (right arrowhead) (magnification: 400x)

\section{Discussion}

To the best of our knowledge, this study was the largest series of IFT in mechanically ventilated critically ill patients. The main findings of our study are as follows.
First, $80.6 \%$ of the patients had proven IFT, whereas the remaining patients (19.4\%) had probable IFT. Second, DM was the most frequent predominant underlying condition in our IFT patients (58.1\%), followed by chronic 
lung disease (38.7\%). Third, less than $15 \%$ of the patients had a hematologic disease. Fourth, none of the patients had neutropenia or had undergone solid organ transplantations. Fifth, the mortality rate was $93.5 \%$. All patients developed acute respiratory failure before diagnosis and were admitted to the ICU with high APACHE II scores $(23.1 \pm 10.4)$.

Invasive fungal infection was reported to mainly affect immunocompromised patients, particularly those with hematologic malignancy [17]. However, Aspergillus spp. has recently been shown to cause invasive fungal disease in patients with chronic lung disease and critically ill patients with liver cirrhosis [7, 19-24]. This is mainly attributed to the administration of broad spectrum antibiotics, corticosteroids, and immunoparalysis related to sepsis [2, 22, 25-27]. In a cohort study of 156 patients with Aspergillus tracheobronchitis, Fernández-Ruiz et al. [15] reported that $6.5 \%$ of them had DM, 23.7\% received mechanical ventilation because of respiratory failure, most patients were immunocompromised, and the mortality rate was $39.1 \%$. Karnak et al. [13] reviewed 228 patients with endobronchial fungal disease and found that $54 \%$ of them were immunocompromised, and $11 \%$ had DM; the mortality rate was $52 \%$ in patients with endobronchial mucormycosis. In our cohort, DM was the most prevalent underlying condition (58.1\%), whereas only four patients $(12.9 \%)$ had hematologic disease, and none of the patients were neutropenic. Numerous studies have reported that acute respiratory failure and ICU admission are crucial prognostic factors for invasive fungal disease $[5,15,27-30]$. In the current study, we found that patients with IFT who developed respiratory failure exhibited high mortality. He et al. [14] reported that patients with Aspergillus tracheobronchitis and the involvement in parenchyma demonstrated higher mortality, suggesting that Aspergillus tracheobronchitis is an early stage of invasive pulmonary aspergillosis. By contrast, Patterson and Strek [2] argued that tracheobronchitis is a form of invasive pulmonary aspergillosis and is associated with poor outcomes because of delayed diagnosis. Furthermore, Karnak et al. [13] also found that 7-20\% of patients with invasive pulmonary aspergillosis simultaneously manifested fatal tracheobronchial involvement. The overall mortality of invasive fungal infection is approximately $50 \%[5,6]$, and the mortalities of endobronchial fungal disease caused by different fungi range widely from 20 to $80 \%$ [11, 13-16]. In our cohort, the overall in-hospital mortality rate was 93.5\% even though none of the patients had neutropenia or immunosuppression. Cornillet et al. suggested that among non-neutropenic patients with invasive aspergillosis, the nonspecific symptoms and the difficulty in diagnosis lead to suboptimal management and the late administration of treatment. This delayed administration of treatment results in a higher mortality in non-neutropenic patients than in neutropenic patients $(89$ vs. $60 \%$ ) and may explain the high mortality observed in our cohort [24, 31].

Diagnosing IFT is considerably difficult because of the nonspecific clinical manifestations and the lack of a diagnostic tool to distinguish colonization from infection $[2,12,32,33]$. Radiological findings are usually nonspecific in patients with aspergillosis. The typical halo and air crescent signs on CT images of the lungs are associated with low sensitivity for invasive fungal infection and are rarely observed in non-neutropenic patients [12, 21]. In the current study, the air crescent sign was detected on the CT scan of only one patient. The galactomannan antigen levels in BAL fluid are useful for diagnosing invasive pulmonary aspergillosis [34]. However, in addition to Aspergillus spp., other fungi may cause invasive tracheobronchitis without affecting the galactomannan antigen levels [13]. Invasive aspergillosis is the most frequent invasive fungal infection among hematologic patients (59.2-74.3\%), followed by invasive fungal infections caused by Mucorales (7.2-13.9\%) [35, 36]. Karnak et al. [13] also found that Aspergillus spp. is the most common pathogenic species of endobronchial fungal disease (53\%), and Mucorales accounted for only $13.4 \%$. In the current study, Aspergillus spp. was the most common pathogenic species (61.3\%), followed by Mucorales (25.8\%).

Considering the increasing incidence of invasive aspergillosis in critically ill patients and the strict host criteria in the revised definitions for invasive fungal disease from the EORTC/MSG [17], Blot et al. [37] validated a clinical algorithm to diagnose invasive pulmonary aspergillosis in the ICU. They proposed Aspergillus-positive lower respiratory tract specimen culture as the entry criterion and that physicians should increasingly consider the possibility of invasive pulmonary aspergillosis [20, 22, 37]. However, the rate of positive fungal culture results for BAL fluid is only $25-77 \%$ and can further decrease after antifungal therapy $[13,31,32]$. In our patients, less than $30 \%$ had positive cultures for fungi before bronchoscopy, and $20 \%$ had negative BAL fluid cultures after bronchoscopy. The incidence of IFT may be underestimated if the diagnosis is based only on positive cultures. Hence, we believe that bronchoscopy should be the main diagnostic approach for IFT.

In 1995, Denning proposed three forms of Aspergillus tracheobronchitis, pseudomembranous, obstructive, and ulcerative [18]. Thereafter, Karnak et al. [13] demonstrated that endobronchial fungal disease can be caused by six different fungi. The origins of invasive fungal diseases are difficult to differentiate on the basis of radiographic or bronchoscopic findings (Fig. 1). Direct 
microscopy of tracheobronchial specimens is essential for observing fungal morphology, enabling a presumptive diagnosis of IFT and earlier administration of antifungal treatment [12]. Transbronchial lung biopsy or bronchial aspiration is relatively risky in patients with angioinvasive fungal infection, particularly in patients with coagulopathy or thrombocytopenia [26, 38]. By contrast, the biopsy of bronchial lesions is less invasive and relatively safe; biopsy exhibits a higher sensitivity than BAL fluid culture alone $[14,16]$. In our study, all patients underwent bronchoscopy and BAL cultures and 25 underwent bronchial biopsy. None of the patients developed massive hemoptysis or hypoxemia after the procedure.

The present study had some limitations. First, this study was retrospective. Second, this study included only cases with proven and probable IFT; thus, some of the possible cases may have been overlooked, and the outcomes may have been underestimated.

\section{Conclusion}

IFT in mechanically ventilated critically ill patients is a devastating disease irrespective of the host's immune status. DM is the most prevalent underlying disease, followed by chronic lung disease. The mortality rate is very high in IFT patients who develop respiratory failure. In addition to Aspergillus spp. (61.3\%), Mucorales is another crucial pathogenic species (25.8\%). Early diagnosis of IFT is based on bronchoscopy. Bronchoscopy with the biopsy of bronchial lesions should be the preferred diagnostic strategy. Large, prospective studies are urgently required to improve the outcome of IFT in critically ill patients.

\section{Abbreviations}

IFT: invasive fungal tracheobronchitis; ICU: intensive care unit; BAL: bronchoalveolar lavage; COPD: chronic obstructive pulmonary disease; APACHE II: Acute Physiology and Chronic Health Evaluation II; CT: computed tomography.

\section{Authors' contributions}

CYL and WLL had full access to all of the data in the study and take responsibility for the integrity of the data and the accuracy of the data analysis. CYL was involved in the study design and contributed to the data collection and writing, review, and revision of the manuscript. WLL was involved in the study design and contributed to the data collection and writing, review, and revision of the manuscript. CCC contributed to the data collection and review of the manuscript. HTC contributed to the data collection and review of the manuscript. $\mathrm{HCH}$ contributed to data interpretation and commented on the manuscript. KK contributed to data collection and commented on the manuscript. NHC supervised data collection and commented on the manuscript. YJC was involved in the study design, supervised statistical analysis, and data interpretation. CTY contributed to data interpretation and commented on the manuscript. GD contributed to data interpretation and commented on the manuscript. CCH provided substantial support to the study design, data analysis, patient recruitment, and manuscript revision. All authors read and approved the final manuscript.

\section{Author details}

1 Department of General Medicine and Geriatrics, Chang Gung Memorial Hospital at Linkou, Taoyuan, Taiwan. ${ }^{2}$ Department of Pulmonary and Critical Care Medicine, Chang Gung Memorial Hospital at Linkou, Taoyuan, Taiwan.
${ }^{3}$ College of Medicine, Chang Gung University, Taoyuan, Taiwan. ${ }^{4}$ Department of Intensive Care Medicine, Chi Mei Medical Center, Liouying, Tainan, Taiwan. ${ }^{5}$ College of Health Sciences, Graduate Institute of Medical Sciences, Chang Jung Christian University, Tainan, Taiwan. ${ }^{6}$ College of Medicine, Fu Jen Catholic University, New Taipei, Taiwan. ${ }^{7}$ Department of Pulmonary and Critical Care Medicine, Chang Gung Memorial Hospital at Chiayi, Chiayi, Taiwan. ${ }^{8}$ Department of Critical Care Medicine, Far Eastern Memorial Hospital, New Taipei City, Taiwan. ${ }^{9}$ Department of Critical Care, ATTIKON University Hospital, University of Athens, Medical School, Athens, Greece.

\section{Acknowledgements}

We thank all of the investigators and members of the Division of Pulmonary and Critical Care Medicine and the Department of Internal Medicine at Chang Gung Memorial Hospital for their efforts.

\section{Competing interests}

The authors declare that they have no competing interests.

Received: 22 August 2016 Accepted: 20 December 2016

Published online: 06 January 2017

\section{References}

1. Limper AH, Knox KS, Sarosi GA, et al. An official American Thoracic Society statement: treatment of fungal infections in adult pulmonary and critical care patients. Am J Respir Crit Care Med. 2011;183:96-128. doi:10.1164/ rccm.2008-740ST.

2. Patterson KC, Strek ME. Diagnosis and treatment of pulmonary aspergillosis syndromes. Chest. 2014;146:1358-68. doi:10.1378/chest.14-0917.

3. Chamilos G, Marom EM, Lewis RE, et al. Predictors of pulmonary zygomycosis versus invasive pulmonary aspergillosis in patients with cancer. Clin Infect Dis. 2005:41:60-6. doi:10.1086/430710.

4. Segal BH. Aspergillosis. N Engl J Med. 2009;360:1870-84. doi:10.1056/ NEJMra0808853.

5. Slavin M, van Hal S, Sorrell TC et al. Invasive infections due to filamentous fungi other than Aspergillus: epidemiology and determinants of mortality. Clin Microbiol Infect. 2015;21: 490 e491-490 e410. doi:10.1016/j. cmi.2014.12.021.

6. Nivoix $Y$, Velten $M$, Letscher-Bru $V$, et al. Factors associated with overall and attributable mortality in invasive aspergillosis. Clin Infect Dis. 2008:47:1176-84. doi:10.1086/592255.

7. Meersseman W, Lagrou K, Maertens J, Van Wijngaerden E. Invasive aspergillosis in the intensive care unit. Clin Infect Dis. 2007;45:205-16. doi:10.1086/518852.

8. Muskett H, Shahin J, Eyres G, et al. Risk factors for invasive fungal disease in critically ill adult patients: a systematic review. Crit Care. 2011;15:R287. doi:10.1186/cc10574.

9. Meersseman W, Vandecasteele SJ, Wilmer A, et al. Invasive aspergillosis in critically ill patients without malignancy. Am J Respir Crit Care Med. 2004;170:621-5. doi:10.1164/rccm.200401-0930C.

10. Ferrell BA, Tolle JJ. Invasive endobronchial mucormycosis. Am J Respir Crit Care Med. 2014;190:e28. doi:10.1164/rccm.201312-2229/M.

11. He H, Ding L, Li F, Zhan Q. Clinical features of invasive bronchial-pulmonary aspergillosis in critically ill patients with chronic obstructive respiratory diseases: a prospective study. Crit Care. 2011;15:R5. doi:10.1186/ cc9402.

12. Bassetti M, Righi E, De Pascale G, et al. How to manage aspergillosis in non-neutropenic intensive care unit patients. Crit Care. 2014;18:458. doi:10.1186/s13054-014-0458-4.

13. Karnak D, Avery RK, Gildea TR, et al. Endobronchial fungal disease: an under-recognized entity. Respiration. 2007;74:88-104. doi:10.1159/000094708.

14. He H, Jiang $S$, Zhang $L$, et al. Aspergillus tracheobronchitis in critically ill patients with chronic obstructive pulmonary diseases. Mycoses. 2014;57:473-82. doi:10.1111/myc.12185.

15. Fernandez-Ruiz M, Silva JT, San-Juan R, et al. Aspergillus tracheobronchitis report of 8 cases and review of the literature. Medicine. 2012;91:261-73. doi:10.1097/MD.0b013e31826c2ccf. 
16. Tasci S, Glasmacher A, Lentini S, et al. Pseudomembranous and obstructive Aspergillus tracheobronchitis_optimal diagnostic strategy and outcome. Mycoses. 2006;49:37-42. doi:10.1111/j.1439-0507.2005.01180.x.

17. De Pauw B, Walsh TJ, Donnelly JP, et al. Revised definitions of invasive fungal disease from the European Organization for Research and Treatment of Cancer/Invasive Fungal Infections Cooperative Group and the National Institute of Allergy and Infectious Diseases Mycoses Study Group (EORTC/MSG) Consensus Group. Clin Infect Dis. 2008;46:1813-21. doi:10.1086/588660.

18. Denning DW. Commentary: unusual manifestations of aspergillosis. Thorax. 1995:50:812-3. doi:10.1136/thx.50.7.812.

19. Leav BA, Fanburg B, Hadley S. Invasive pulmonary aspergillosis associated with high-dose inhaled fluticasone. N Engl J Med. 2000;343:586. doi:10.1056/NEJM200008243430818.

20. Garbino J, Fluckiger U, Elzi L, et al. Survey of aspergillosis in nonneutropenic patients in Swiss teaching hospitals. Clin Microbiol Infect. 2011;17:1366-71. doi:10.1111/j.1469-0691.2010.03402.x.

21. Guinea J, Torres-Narbona M, Gijon P, et al. Pulmonary aspergillosis in patients with chronic obstructive pulmonary disease: incidence, risk factors, and outcome. Clin Microbiol Infect. 2010;16:870-7. doi:10.1111/j.1469-0691.2009.03015.x.

22. Dimopoulos G, Frantzeskaki F, Poulakou G, Armaganidis A. Invasive aspergillosis in the intensive care unit. Ann N Y Acad Sci. 2012;1272:31-9. doi:10.1111/j.1749-6632.2012.06805.x.

23. Delsuc C, Cottereau A, Frealle E, et al. Putative invasive pulmonary aspergillosis in critically ill patients with chronic obstructive pulmonary disease: a matched cohort study. Crit Care. 2015;19:421. doi:10.1186/ s13054-015-1140-1.

24. Cornillet A, Camus C, Nimubona S, et al. Comparison of epidemiological, clinical, and biological features of invasive aspergillosis in neutropenic and nonneutropenic patients: a 6-year survey. Clin Infect Dis. 2006;43:577-84. doi:10.1086/505870.

25. Hotchkiss RS, Monneret G, Payen D. Immunosuppression in sepsis: a novel understanding of the disorder and a new therapeutic approach. Lancet Infect Dis. 2013;13:260-8. doi:10.1016/S1473-3099(13)70001-X.

26. Kosmidis C, Denning DW. The clinical spectrum of pulmonary aspergillosis. Thorax. 2015;70:270-7. doi:10.1136/thoraxjnl-2014-206291.

27. Taccone FS, Van den Abeele AM, Bulpa P, et al. Epidemiology of invasive aspergillosis in critically ill patients: clinical presentation, underlying conditions, and outcomes. Crit Care. 2015;19:7. doi:10.1186/ s13054-014-0722-7.

28. Kennedy KJ, Daveson K, Slavin MA, et al. Mucormycosis in Australia: contemporary epidemiology and outcomes. Clin Microbiol Infect. 2016; doi:10.1016/j.cmi.2016.01.005.

29. Spellberg B, Kontoyiannis DP, Fredricks D, et al. Risk factors for mortality in patients with mucormycosis. Med Mycol. 2012;50:611-8. doi:10.3109/136 93786.2012.669502.

30. Vandewoude K, Blot S, Benoit D, et al. Invasive aspergillosis in critically ill patients: analysis of risk factors for acquisition and mortality. Acta Clin Belg. 2004:59(5):251-7.

31. Bulpa P, Dive A, Sibille Y. Invasive pulmonary aspergillosis in patients with chronic obstructive pulmonary disease. Eur Respir J. 2007;30:782-800. doi:10.1183/09031936.00062206.

32. Panigrahi MK, Manju R, Kumar SV, Toi PC. Pulmonary mucormycosis presenting as nonresolving pneumonia in a patient with diabetes mellitus. Respir Care. 2014;59:e201-5. doi:10.4187/respcare.03205.

33. Wang XM, Guo LC, Xue SL, Chen YB. Pulmonary mucormycosis: a case report and review of the literature. Oncol Lett. 2016;11:3049-53. doi:10.3892/ol.2016.4370.

34. Meersseman W, Lagrou K, Maertens J, et al. Galactomannan in bronchoalveolar lavage fluid: a tool for diagnosing aspergillosis in intensive care unit patients. Am J Respir Crit Care Med. 2008;177:27-34. doi:10.1164/ rccm.200704-6060C.

35. Klingspor L, Saaedi B, Ljungman P, Szakos A. Epidemiology and outcomes of patients with invasive mould infections: a retrospective observational study from a single centre (2005-2009). Mycoses. 2015;58:470-7. doi:10.1111/myc.12344.

36. Neofytos D, Horn D, Anaissie E, et al. Epidemiology and outcome of invasive fungal infection in adult hematopoietic stem cell transplant recipients: analysis of Multicenter Prospective Antifungal Therapy (PATH) Alliance registry. Clin Infect Dis. 2009;48:265-73. doi:10.1086/595846.

37. Blot SI, Taccone FS, Van den Abeele AM, et al. A clinical algorithm to diagnose invasive pulmonary aspergillosis in critically ill patients. Am J Respir Crit Care Med. 2012;186:56-64. doi:10.1164/rccm.201111-19780C.

38. Di Carlo P, Cabibi D, La Rocca AM, et al. Post-bronchoscopy fatal endobronchial hemorrhage in a woman with bronchopulmonary mucormycosis: a case report. J Med Case Rep. 2010;4:398. doi:10.1186/1752-1947-4-398.

\section{Submit your manuscript to a SpringerOpen ${ }^{\odot}$ journal and benefit from:}

- Convenient online submission

- Rigorous peer review

- Immediate publication on acceptance

- Open access: articles freely available online

- High visibility within the field

- Retaining the copyright to your article

Submit your next manuscript at springeropen.com 